\title{
Stereochemical analysis of $(+)$-limonene using theoretical and experimental NMR and chiroptical data
}

\author{
F. Reinscheid, U.M. Reinscheid* \\ NMR-Based Structural Biology, Max-Planck-Institute of Biophysical Chemistry, Am Fassberg 11, 37077 Göttingen, Germany
}

\section{A R T I C L E I N F O}

\section{Article history:}

Received 10 July 2015

Received in revised form

15 September 2015

Accepted 19 October 2015

Available online 24 October 2015

\section{Keywords:}

Limonene

ORD

ECD

VCD

NMR

Conformation

\begin{abstract}
A B S T R A C T
Using limonene as test molecule, the success and the limitations of three chiroptical methods (optical rotatory dispersion (ORD), electronic and vibrational circular dichroism, ECD and VCD) could be demonstrated. At quite low levels of theory (mpw1pw91/cc-pvdz, IEFPCM (integral equation formalism polarizable continuum model)) the experimental ORD values differ by less than 10 units from the calculated values. The modelling in the condensed phase still represents a challenge so that experimental NMR data were used to test for aggregation and solvent-solute interactions. After establishing a reasonable structural model, only the ECD spectra prediction showed a decisive dependence on the basis set: only augmented (in the case of Dunning's basis sets) or diffuse (in the case of Pople's basis sets) basis sets predicted the position and shape of the ECD bands correctly. Based on these result we propose a procedure to assign the absolute configuration (AC) of an unknown compound using the comparison between experimental and calculated chiroptical data.
\end{abstract}

() 2015 Elsevier B.V. All rights reserved.

\section{Introduction}

In the field of configurational analysis it is very important to use structural information since only then a reliable assignment of the absolute configuration of new molecules is possible [1,2]. Despite enormous progress in instrumentation and methods [3] structural analysis is still a challenge, illustrated by the review of Nicolaou and Snyder [4] who presented data based on which roughly 1000 articles published between 1990 and 2004 have to be revised because of structural reasons. Likewise, Maier [5] presented numerous recent examples of erroneous chemical formulas. One of the early configurational assignment strategies is based on the chiral recognition of unsaturated compounds with optically active lanthanide complexes measured by NMR. Offermann and Mannschreck [6] used limonene to demonstrate that the changes in chemical shifts by titrating the analyte with chiral lanthanide complexes can be used to determine the enantiomeric excess.

The chiral monoterpene limonene is an important starting material in chemical synthesis [7-9]. Moreover, it is used in the fragrance and food industry, and is an important synthetic material [10]. Limonene is biotransformed by a variety of organisms such as

\footnotetext{
* Corresponding author.

E-mail address: urei@nmr.mpibpc.mpg.de (U.M. Reinscheid).
}

bacteria, fungi, and plants [11]. In this way, using appropriate species a number of synthetically important metabolites can be produced. One of the biosynthetic enzymes, limonene epoxide hydrolase, was recently used as test case for asymmetric biocatalysis [12].

Limonene has served as chiral test molecule to investigate chiroptical methods such as vibrational circular dichroism (VCD), electronic circular dichroism (ECD), and optical rotatory dispersion (ORD), and even as inducer of chirality. After the early report of Noack [13] using the monoterpene menthol, Kobayashi et al. [14], Fukiji et al. [15a] and Aimi et al. [15b] were able to induce optical activity in achiral environments by limonene enantiomers. A review summarizes solvent chirality transfer in the field of supramolecular chemistry [15c]. In recent publications, the orientation of limonene enantiomers was measured by sum frequency vibrational spectroscopy, detecting surface chirality [16].

In our report, we present a detailed analysis of spectroscopic data about (+)-limonene in order to (i) deliver detailed analytical data about the academically and industrially important monoterpene limonene and (ii) improve the methodology of chiral analysis. Based on the results for strychnine base and the protonated $/ \mathrm{HCl}$ form $[17,18]$, we further explore the success and limitations of the absolute configuration (AC) determination using a comparison of experimental and calculated chiroptical data. Two recent publications nicely illustrate the importance of solvent 
modelling for the interpretation of chiroptical data [19a]. Consequently, we will discuss the influence of solvent models on the determination of the absolute configuration.

NMR and chiroptical data about limonene are combined, and all three major chiroptical methods (VCD, ECD, ORD) are applied. The importance to use more than one chiroptical method to determine the absolute configuration of a compound has been convincingly put forward by Polavarapu [19b]. As an example, the AC of two nonylphenols was determined by optical rotation and VCD analysis [20]. For one of the compounds (NP112) it is still unclear if the AC was correctly assigned due to impurity problems. The AC assignment of nonylphenol NP35 [20] was confirmed by x-ray analysis [21].

The present report is organized in the following way: First, the structural models of the relevant conformers are obtained together with their populations; second, population-weighted chiroptical data are calculated for the structural models and compared to experimental values; third, conclusions derived from these comparisons are critically assessed.

\section{Results}

In order to develop a general procedure to analyse the configuration of compounds for which NMR data are available, limonene is a good choice since it serves as test molecule for NMR method development. For chemical shifts we used information from Skakovskii et al. [22] and Bohlmann et al. [23] In addition, deuterium NMR helped in assigning diastereotopic protons [24]. Assuming similar trends for both nuclei, protons and deuteriums, the calculations unambiguously assigned the methylene protons of limonene.

\subsection{Structural models}

Limonene represents a simple monoterpene (Fig. 1). At first sight, it might be conformationally flexible in the six-membered ring. The conformational search (Discovery Studio, Accelrys [25], Universal forcefield) of a model for $R$-limonene delivered two types of ring conformers which differed in the orientation of the isopropenyl substituent: equatorial or axial. However, the axial orientation of the isopropenyl substituent raises the computed energy substantially. Since the energy difference to the conformers with an equatorial isopropenyl substituent was larger than $2.5 \mathrm{kcal} /$ mol, we can safely assume that almost exclusively the latter form exists in solution. Therefore, conformational averaging due to ring inversions cannot be taken as explanations in the course of spectrum interpretation [24]. It is more likely, that the rotation of the isopropenyl group is responsible for averaged signals in NMR spectra.

Having established the correct form with the isopropenyl group in the equatorial position, a full systematic conformational search

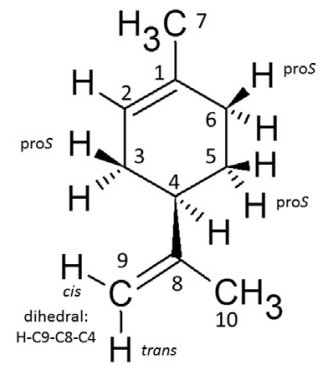

Fig. 1. (+)-R-limonene. was conducted using Discovery Studio. With the applied universial forcefield ten possible conformers were obtained with a cut-off of $2.5 \mathrm{kcal} / \mathrm{mol}$ higher than the lowest energy conformer. All ten were further geometry optimized with Gaussian09 [26] (mpw1pw91/ccpvdz; IEF-PCM: chloroform). During this step, only three optimized conformers remained (Fig. 2A-D) indicating that most often nonstable conformations were generated in the forcefield search. Frequency calculations confirmed that all three are minimum structures due to the absence of vibrations with imaginary frequencies. The ring dihedrals only differ slightly among the isopropenyl rotamers, with differences smaller than $5^{\circ}$ (Table 1$)$. The isopropenyl dihedrals are similar (differences smaller than $4^{\circ}$ ) to the dominant conformers calculated by Debie: [27] $+132,-142$, and -8 for conformer 1,2 and 3, respectively, which were obtained on the B3LYP/6-31g(d) level of theory in vacuo. For comparison, the axial conformer at lowest energy is also shown in Table 1 . It is $1.9 \mathrm{kcal} /$ mol higher in free energy compared to the highest in energy equatorial conformer (conformer 1) calculated at the mpw1pw91/ cc-pvdz; IEF-PCM: chloroform level of theory.

\subsection{Population analysis}

We followed two approaches for population analysis starting with the purely computational one. The three conformers differed in free energies depending on which level of theory and which solvent was used for modelling. Different levels of theory and two solvents (ethanol and chloroform) were used for frequency calculations and the free energy values are presented in Table 2. Using the Boltzmann equation we calculated the populations for the three conformers (Table 3). Since data with neat limonene have to be interpreted, it is important not to use only one solvent that might be an inappropriate substitute for limonene as solvent. So two largely different solvents and two different basis sets were used and the arithmetic mean for the calculated populations based on free energies were determined for conformer 1,2 , and 3 as $28 \%, 38 \%$, and $34 \%$, respectively. The standard deviations of the calculated populations are $3 \%, 6 \%$, and $3 \%$, respectively, which fits nicely to results from literature e.g. glucose [28]; endo-borneol [29]; cylohexene oxides [30]. Interestingly, the calculated entropies are highest for conformer 2 at all levels of theory (Table 2).

Populations derived from calculated enthalpies differ (Tables 2, and 3 ) in that conformer 2 is less populated due to the neglect of entropic contributions (34:23:43). Since calculated entropies might be unreliable due to a large error associated with vibrations at low wavenumbers $\left(<200 \mathrm{~cm}^{-1}\right)$ it is not easy to decide if free energies or enthalpies deliver accurate energies for the population analysis. Applying a reasonable scaling factor in the thermochemical analysis does not solve the problem. Based on the literature [31-33] we can assume, that for the levels of theory in this study (mpw1pw91 as a functional, and the Dunning's basis sets) an average scaling factor of 0.97 can be assumed. Please note that Irikura et al. [34] suggested that only two digits are meaningful. As an example, using a scaling factor to zero-point vibrational energies and thermal corrections to enthalpies of 0.97 , gives for the mpw1pw91/aug-ccpvtz/IEFPCM (chloroform) level of theory the following populations for conformer 1,2 , and $3: 31 \%, 28 \%$, and $41 \%$, which differ to the values obtained using unscaled free energies or enthalpies.

The second approach for the population analysis represents a combination of experimental values and calculated data. Based on the three structural models for the conformers, we set out to calculate ${ }^{13} \mathrm{C}$ chemical shifts. Experimental data are readily available from literature which is methodologically important since we want to draw conclusions from this study for the analysis of other molecules for which published values are available. In case of unstable molecules, or natural products for which no natural sources 

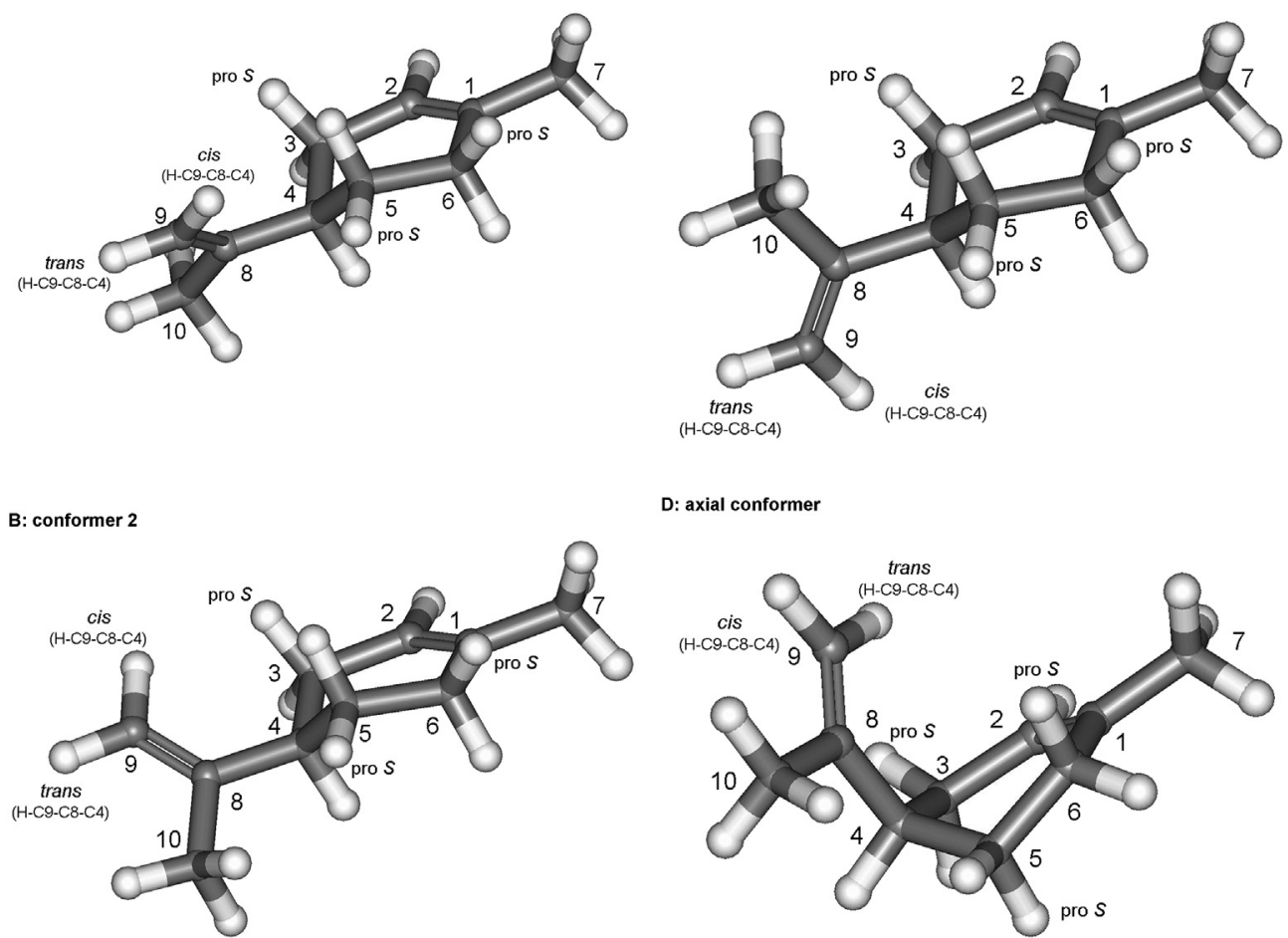

Fig. 2. A-D: Geometry-optimized (mpw1pw91/cc-pvdz, IEFPCM: chloroform) conformers.

for re-isolation are available, it is then still possible to determine populations and to perform a configurational analysis.

The ${ }^{13} \mathrm{C}$ chemical shifts were used for the population analysis. There are three reasons for it: 1 ) it is an established parameter for discriminating isomers and conformers [1];2) The span between the different conformers is quite large for ${ }^{13} \mathrm{C}$ chemical shifts compared to proton chemical shifts; 3 ) For the following analysis it is very important to have enough data for calibration and prediction. If the two steps (calibration and prediction) are only based on few data, the results will become inaccurate. Three levels of theory were used, all three using the IEF-PCM solvent model with chloroform as solvent: theory level 1:mpw1pw91/cc-pvdz; theory level 2: B3LYP/6-311 + g(d,p); theory level 3: PBEPBE/cc-pvtz.

Tables $\mathrm{S} 1-3$ (see supporting information) show the calculated data for the three conformers calculated on the three levels of theory. We first calibrated our calculated data in the following way: we selected resonances that did not differ much among the three conformers (cut-off value was $\leq 1.15 \mathrm{ppm}$ ). For the ${ }^{13} \mathrm{C}$ resonances fulfilling the requirement a linear regression with experimental values as $\mathrm{x}$ component was conducted. As y values the arithmetic mean of the shieldings of the three conformers were taken. The best fit to experiment was possible with theory level 1 (mpw1pw91/cc-pvdz) (Table 4) so that these calibration parameters ( $\mathrm{a}$ and $\mathrm{b}$ from $\mathrm{y}=\mathrm{ax}+\mathrm{b}$ ) were used (Fig. S4A and B, supporting information). The obtained parameters were then used to predict the experimental ${ }^{13} \mathrm{C}$ chemical shifts of the remaining nuclei which showed differences between the conformers larger than $3 \mathrm{ppm}$. From these predicted data, we obtained by a systematic variation of populations a best fit with experimental values with the following population mix: conformer 1,2 , and 3: $39 \%, 31 \%$, and $30 \%$. The sum of CMAE (corrected mean absolute error; sum of absolute differences between calculated (using the linear regression) and experimental values, divided by the number of data pairs) and the variance was taken as indicator of the fit. The lowest sum of these values corresponds to the mentioned population mix which reproduced the experimental data at best. The populations of the experimental based approach are within $\pm 10 \%$ of the populations derived from the pure computational approach.

Table 1

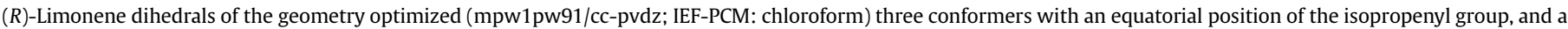
geometry optimized conformer with an axial position of the isopropenyl group.

\begin{tabular}{|c|c|c|c|c|}
\hline Dihedral & Axial & Equatorial conformer 1 & Equatorial conformer 2 & Equatorial conformer 3 \\
\hline $\mathrm{C} 9-\mathrm{C} 8-\mathrm{C} 4-\mathrm{H} 4^{\mathrm{a}}$ & -134.16 & 129.52 & -138.75 & -8.03 \\
\hline $\mathrm{H} 5$ proS-C5-C6-H6proR & -50.94 & 47.11 & 45.73 & 46.68 \\
\hline H5proR-C5-C6-H6proR & 65.63 & 163.99 & 162.68 & 163.75 \\
\hline $\mathrm{H} 5$ proR-C5-C4-H4 & -59.71 & 174.15 & 173.75 & 173.92 \\
\hline $\mathrm{H} 5$ proS-C5-C4-H4 & 56.51 & -68.62 & -69.47 & -68.75 \\
\hline $\mathrm{H} 3$ pro $R-C 3-\mathrm{C} 4-\mathrm{H} 4$ & 78.29 & 166.02 & 170.99 & 166.95 \\
\hline $\mathrm{H} 3$ proS-C3-C4-H4 & -36.23 & 50.62 & 55.16 & 51.54 \\
\hline $\mathrm{H} 3$ proR-C3-C2-H2 & -43.25 & -71.93 & -74.95 & -72.12 \\
\hline H3proS-C3-C2-H2 & 71.21 & 43.07 & 40.06 & 42.86 \\
\hline
\end{tabular}

\footnotetext{
${ }^{a}$ Isopropenyl dihedral.
} 
Table 2

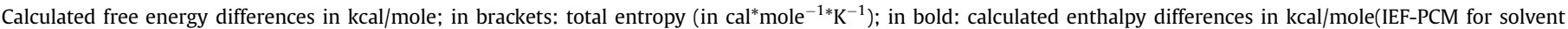
modelling).

\begin{tabular}{|c|c|c|c|c|}
\hline Theory level & Solvent & Conformer 1 & Conformer 2 & Conformer 3 \\
\hline mpw1pw91/cc-pvdz & Chloroform & $\begin{array}{l}0.097 \\
(100.5) \\
\mathbf{0 . 1 3 5}\end{array}$ & $\begin{array}{l}0.113 \\
(101.3) \\
\mathbf{0 . 3 6 6}\end{array}$ & $\begin{array}{l}0 \\
(100.3) \\
\mathbf{0}\end{array}$ \\
\hline mpw1pw91/aug-cc-pvdz & Chloroform & $\begin{array}{l}0.246 \\
(100.0) \\
\mathbf{0 . 1 3 6}\end{array}$ & $\begin{array}{l}0 \\
(101.8) \\
\mathbf{0 . 3 8 3}\end{array}$ & $\begin{array}{l}0.078 \\
(100.1) \\
\mathbf{0}\end{array}$ \\
\hline mpw1pw91/aug-cc-pvtz & Chloroform & $\begin{array}{l}0.284 \\
(100.3) \\
\mathbf{0 . 1 4 2}\end{array}$ & $\begin{array}{l}0 \\
(102.0) \\
\mathbf{0 . 3 5 5}\end{array}$ & $\begin{array}{l}0.134 \\
(100.3) \\
\mathbf{0}\end{array}$ \\
\hline mpw1pw91/cc-pvdz & Ethanol & $\begin{array}{l}0.096 \\
(100.6) \\
\mathbf{0 . 1 3 6}\end{array}$ & $\begin{array}{l}0.093 \\
(101.4) \\
\mathbf{0 . 4 0 3}\end{array}$ & $\begin{array}{l}0 \\
(100.4) \\
\mathbf{0}\end{array}$ \\
\hline mpw1pw91/aug-cc-pvdz & Ethanol & $\begin{array}{l}0.334 \\
(100.1) \\
\mathbf{0 . 1 4 1}\end{array}$ & $\begin{array}{l}0 \\
(102.1) \\
0.405\end{array}$ & $\begin{array}{l}0.205 \\
(100.0) \\
\mathbf{0}\end{array}$ \\
\hline mpw1pw91/aug-cc-pvtz & Ethanol & $\begin{array}{l}0.320 \\
(100.4) \\
\mathbf{0 . 1 4 4}\end{array}$ & $\begin{array}{l}0 \\
(102.2) \\
0.423\end{array}$ & $\begin{array}{l}0.181 \\
(100.4) \\
\mathbf{0}\end{array}$ \\
\hline
\end{tabular}

Table 3

Populations (in \%) based on the Boltzmann equation using free energy differences, in brackets: enthalpy differences (IEF-PCM for solvent modelling).

\begin{tabular}{|c|c|c|c|c|}
\hline Theory level & Solvent & Conformer 1 & Conformer 2 & Conformer 3 \\
\hline mpw1pw91/cc-pvdz & Chloroform & $32(34)$ & $31(23)$ & $37(43)$ \\
\hline mpw1pw91/aug-cc-pvdz & Chloroform & $26(34)$ & $39(23)$ & $35(43)$ \\
\hline mpw1pw91/aug-cc-pvtz & Chloroform & $26(34)$ & $41(23)$ & $33(43)$ \\
\hline mpw1pw91/cc-pvdz & Ethanol & $31(35)$ & $32(22)$ & $37(43)$ \\
\hline mpw1pw91/aug-cc-pvdz & Ethanol & $25(34)$ & $44(22)$ & $31(44)$ \\
\hline mpw1pw91/aug-cc-pvtz & Ethanol & $25(34)$ & $43(22)$ & $32(44)$ \\
\hline
\end{tabular}

Table 4

Linear regression parameters.

\begin{tabular}{|c|c|c|c|}
\hline Level of theory & Slope/standard error & Intercept/standard error & Regression coefficient $\mathrm{R}^{2}$ \\
\hline mpw1pw91/cc-pvdz & $-1.0092 / 0.002$ & $195.27 / 0.23$ & 0.99999 \\
\hline B3LYP/6-311 + g(d,p) & $-1.0759 / 0.015$ & $182.45 / 1.54$ & 0.99946 \\
\hline PBEPBE/cc-pvtz & $-1.0416 / 0.013$ & $181.75 / 1.40$ & 0.99522 \\
\hline
\end{tabular}

Using the population mix of 39:31:30, the resonances with differences between the conformers of $>1.15$ ppm were predicted and compared to experiment (Table 5 ). The very good match supports the view that the populations were accurately determined. All five resonances differ by less than $1 \mathrm{ppm}$ between experimental and prediction. The corrected mean absolute error (CMAE) is $0.47 \mathrm{ppm}$. With the calculated populations (free energies, 28:38:34 and enthalpies 34:23:43) the CMAE is increased to 0.52 and 0.61 , respectively. These values benefit from the internal referencing so that typical regression formulae based on a variety of compounds give much larger errors, e.g. $2.07 \mathrm{ppm}$ of a mean unsigned error on the B3LYP/6-311 $+\mathrm{g}(\mathrm{d}, \mathrm{p}) / / 6-31 \mathrm{~g}(\mathrm{~d})$ level of theory (van Eikema Hommes and Clark, 2005) [35].

Depending on the level of theory, calculated populations differ.

\section{Table 5}

Predicted (mpw1pw91/cc-pvdz; IEF-PCM using chloroform as solvent), populationweighted (39:31:30) and experimental carbon chemical shifts.

\begin{tabular}{lcc}
\hline Resonance & Experimental & Predicted \\
\hline C3 & 31.5 & 31.8 \\
C4 & 41.8 & 42.4 \\
C5 & 28.6 & 29.1 \\
C9 & 109.1 & 108.1 \\
C10 & 21.5 & 20.9 \\
\hline
\end{tabular}

Debie [27] reported 95.2\% equatorial and 4.8\% axial forms (B3LYP/6$31 \mathrm{~g}(\mathrm{~d})$, in vacuo) with respect to the isopropenyl group. Importantly, it was argued that the B3LYP functional gives unreliable energy differences for six-membered rings [36]. We did not find any axial conformer contributing substantially to the population mix, i.e. the lowest in energy was $1.9 \mathrm{kcal} / \mathrm{mol}$ higher in energy compared to the highest in energy of the three equatorial conformers.

The calculated populations of the equatorial conformers [27] differ from ours although the structures are quite similar (see above). For the corresponding equatorial conformers 1, 2, and 3 the populations were $31.1,19.5$, and 44.6 . It was concluded that some bands in the IR and VCD spectra cannot be assigned, probably due to combination bands and overtones [27]. However, inaccurate populations could also be the reason since these were calculated in vacuo, but applied to simulate spectra obtained in solution. In addition, taking the populations from Debie [27] we obtained a worse match between experimental and predicted carbon resonances.

Under vacuum, reliable population predictions based on calculations might be easier. Śmiałek et al. [37] calculated a population mix for limonene of 55:29:16 (all with the equatorial position of the isopropenyl group; theory levels: geometry: MP2/cc-pvdz; energy: CCSD/cc-pvdz; all calculations in vacuo). Vacuum UV spectroscopy 
results agreed well with the calculated absorption frequencies.

We can conclude that literature data based on a pure computational approach differ significantly. Due to the importance of accurate and precise populations, we recommend a combined approach of experimental and computed data such as ${ }^{13} \mathrm{C}$ resonances.

\subsection{Single point at $589 \mathrm{~nm}:[\alpha]_{D}$}

To assess the precision of the calculated ORD data, we applied several levels of theory for calculating the optical rotation of $(+)-(R)$-limonene assuming a 39:31:30 population mix. These values together with relative computational times are listed in Table 6. The arithmetic mean (seven levels of theory) of $[\alpha]_{D}$ is 119.5 with a standard deviation of 4.8 which matches very well to the experimental value of 113.8 (Table 7). On top of these uncertainties, the population mix might change up to $\pm 5 \%$ so that in extreme cases (34:36:30 and 44:26:30), the mean values are 96.8 and 142.8 and the standard deviations are 6.9 and 3.3. Using the populations based on free energies (28:38:34) the arithmetic mean and standard deviation amount to 71.2 (9.4), and with enthalpies (34:23:43) to 103.6 (5.5). Interestingly, only using the calculated optical rotation values, the best match with experiment $\left([\alpha]_{D}\right.$ of pure limonene: 122.8; [38]) is obtained with a population mix of 40:30:30, very close to the mix obtained using experimental carbon chemical shifts. It remains unclear why Leopold et al. [39] measured a $[\alpha]_{D}$ value of +96.6 for the neat compound at $21{ }^{\circ} \mathrm{C}$ with a $98 \%$ purity assessed by GC.

It is important to look at the absolute values since we can assume from literature that low experimental $[\alpha]_{D}$ values in the range from -10 to +10 may have an incorrect sign. When analysing natural products with a low enantiomeric excess other optically active impurities might change the absolute value and sign of $[\alpha]_{D}$. In the case of limonene with absolute values in the range of 100 , the experimentally determined sign is safe. The dependence of the calculated optical rotation on the population mix clearly limits the assignment of absolute configurations to cases with experimental absolute $[\alpha]_{D}$ values larger than roughly 10 . This consists of (i) $\pm 5 \%$ population error which translates for $[\alpha]_{D}=100$ to $\pm \Delta[\alpha]_{D}=5$ and (ii) on top a computational error of \pm 5 in calculating the specific optical rotation.

In order to compare the calculated uncertainties, experimental $[\alpha]_{D}$ data from literature [40] in different solvents are shown in Table 7. From these data a mean value of 113.5 and a standard deviation of 2.7 are calculated. In addition, using different solvents in the continuum model, similar differences are obtained (Table 8). The mean value is 114.4 and the standard deviation is 2.1. Therefore, it is reasonable to assume similar levels of precision for the solvent influence on $[\alpha]_{D}$ for the experimental and calculated data and the match between experiment and calculation is excellent. Consequently, the comparison between two different solvents is justified since this would introduce an error of $5 \%$ at maximum.

In addition, we checked the influence of the solvent used in the
Table 7

Experimental $[\alpha]_{D}$ values of $(+)$-limonene in different solvents at $20{ }^{\circ} \mathrm{C}$ [40], corrected by using the experimental value of Wilson et al. [38] for an homogenous sample (122.8) (experimental value for homogenous limonene was 124.2 by Rule and Chambers [40].

\begin{tabular}{ll}
\hline Solvent & {$[\alpha]_{\mathrm{D}}$} \\
\hline Methanol & 108.5 \\
Acetonitrile & 109.4 \\
Acetone & 112.1 \\
Chloroform & 113.8 \\
Cyclohexane & 114.6 \\
Dichloromethane & 114.7 \\
Homogenous & 122.8 \\
\hline
\end{tabular}

geometry optimization in combination with a solvent change in the calculation of optical rotation at $589 \mathrm{~nm}$ of the three conformers (last four entries in Table 8 ). The specific optical rotation differed by $4.1(4 \%)$ at maximum.

To sum up, we can safely assume, that the structural models with a continuum model for solvent incorporation (IEF-PCM) are appropriate to be used for the prediction of optical rotation values of limonene.

To further broaden the applicability and to increase the reliability of the determination of the absolute configuration using ORD data, a multiple wavelength analysis was performed.

\subsection{Multiple wavelengths ORD}

The calculated values at different wavelengths (Table 9, Fig. S5A supporting information) indicate a mono-signate curve down to $210 \mathrm{~nm}$ which can be easily and safely interpreted. The experimental values in chloroform as solvent are obtained at four wavelengths and are shown together with the population-weighted calculated values (Fig. 3). The experimental values can be used for comparison with the calculated values using methanol as solvent since we have demonstrated that the calculated values are quite insensitive for different solvents (see above), and the experimental values are only slightly affected by different solvents (see above). Calculated and experimental ORD values fit much better (Fig. 3) with the populations derived from experimental and calculated ${ }^{13} \mathrm{C}$ chemical shifts compared to the pure computationally derived populations.

In addition, experimental values obtained from the gas phase at $365 \mathrm{~nm}$ and $633 \mathrm{~nm}$, fit very well to the calculated data in vacuo (Table S5C, supporting information). The calculated data in Fig. 3 directly follow the experimental values in magnitude and sign. If the wavelengths and/or the rotational strengths of the cotton effects are largely inaccurate and at the same time inverted, the assignment of the absolute configuration based on the optical rotation dispersion shown in Fig. 3 would be incorrect. Since these conditions are very unlikely the AC of limonene can be derived from the ORD analysis. We can safely conclude that $(+)$ limonene has $R$ configuration.

Table 6

Calculated $[\alpha]_{\mathrm{D}}$ values of $(+)$-limonene (IEFPCM: chloroform) at different levels of theory using a population mix of 39:31:30 together with relative computational times.

\begin{tabular}{|c|c|c|c|}
\hline Number of theory level & Theory level & Relative computational time & ORD at $589 \mathrm{~nm}$ with $39: 31: 30$ population mix \\
\hline 1 & mpw1pw91/cc-pvdz & 1 & 111.4 \\
\hline 2 & mpw1pw91/aug-cc-pvdz & 4 & 124.4 \\
\hline 3 & mpw1pw91/cc-pvtz & 8 & 119.6 \\
\hline 4 & mpw1pw91/aug-cc-pvtz & 60 & 126.2 \\
\hline 5 & b3lyp/6-311 + g(d,p) & 1 & 121.4 \\
\hline 6 & b3lyp/6-311++g(2d,2p) & 4 & 115.1 \\
\hline 7 & b3lyp/aug-cc-pvdz & 4 & 118.6 \\
\hline
\end{tabular}


Table 8

Calculated (mpw1pw91/cc-pvdz) $[\alpha]_{\mathrm{D}}$ values of $(+)$-limonene in different solvents (IEF-PCM solvent model).

\begin{tabular}{|c|c|c|c|c|}
\hline Solvents & Conformer 1 & Conformer 2 & Conformer 3 & With populations of $39: 31: 30$ \\
\hline Methanol & 432.4 & -141.3 & -41.6 & 112.4 \\
\hline Acetone & 430.7 & -136.5 & -41.9 & 113.1 \\
\hline Dichloro-methane & 427.1 & -126.5 & -42.7 & 114.5 \\
\hline Toluene & 423.7 & -117.0 & -44.7 & 115.6 \\
\hline Benzene & 423.5 & -116.3 & -44.8 & 115.7 \\
\hline Chloroben-zene & 422.2 & -112.4 & -43.3 & 116.8 \\
\hline Aniline & 419.4 & -104.2 & -43.0 & 118.4 \\
\hline Ethanol (geo:eth) & 431.1 & -136.2 & -42.0 & 113.3 \\
\hline Ethanol (geo: $\mathrm{CHCl}_{3}$ ) & 430.7 & -136.5 & -42.3 & 113.0 \\
\hline $\mathrm{CHCl}_{3}$ (geo: eth) & 426.3 & -124.0 & -44.1 & 114.6 \\
\hline $\mathrm{CHCl}_{3}\left(\right.$ geo: $\left.\mathrm{CHCl}_{3}\right)$ & 426.6 & -136.2 & -42.5 & 111.4 \\
\hline
\end{tabular}

Geo: geometry-optimization; eth: ethanol.

Table 9

Calculated ORD values of (+)-limonene at different wavelengths using DFT (mpw1pw91/cc-pvdz, IEFPCM (methanol).

\begin{tabular}{|c|c|c|c|c|}
\hline Wavelength [nm] & Conformer 1 & Conformer 2 & Conformer 3 & Population mix 39:31:30 \\
\hline 589 & 432.4 & -141.3 & -41.6 & 112.4 \\
\hline 578 & 450.8 & -152.9 & -44.7 & 115.0 \\
\hline 546 & 516.0 & -161.7 & -52.5 & 135.4 \\
\hline 495 & 655.6 & -202.2 & -70.3 & 171.9 \\
\hline 436 & 908.6 & -272.9 & -106.0 & 238.0 \\
\hline 365 & 1501 & -427.9 & -206.3 & 391.2 \\
\hline 300 & 2900 & -754.9 & -522.5 & 740.2 \\
\hline 280 & 3815 & -951.1 & -782.7 & 958.5 \\
\hline 265 & 4869 & -1165 & -1129 & 1199 \\
\hline 254 & 5991 & -1384 & -1548 & 1443 \\
\hline 235 & 9331 & -1995 & -3083 & 2095 \\
\hline 220 & 15034 & -2950 & -6662 & 2950 \\
\hline 210 & 23055 & -4126 & -13930 & 3533 \\
\hline 200 & 43171 & -5457 & -52063 & -473.9 \\
\hline 190 & 89442 & -26453 & 33285 & 36667 \\
\hline 180 & -5907213 & -36973 & 631678 & -2125771 \\
\hline 170 & 1603338 & -567424 & -369051 & 338685 \\
\hline 160 & -154568 & -1350916 & 101897 & -448496 \\
\hline
\end{tabular}

\section{5. $U V-V i s / E C D$}

A number of levels of theory were applied for the calculation of rotational strengths of cotton effects (Fig. 4). Based on the calculated rotational strengths of the 10 excitations lowest in energy and

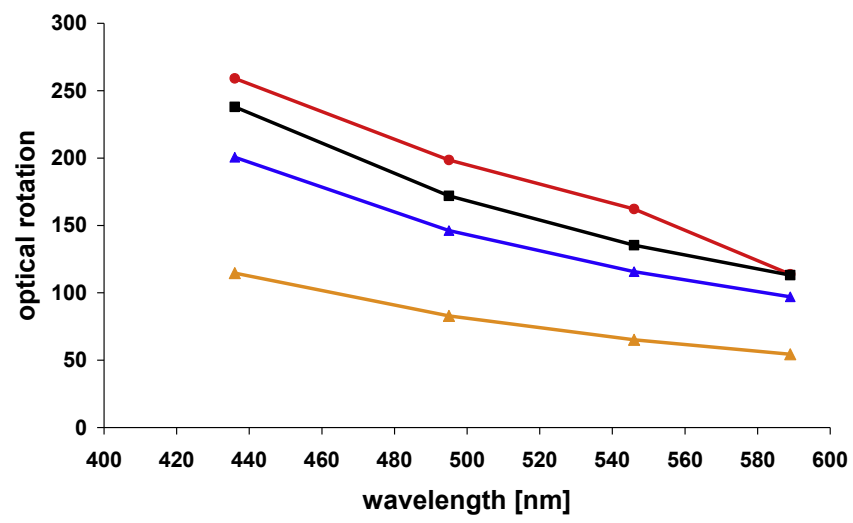

Fig. 3. Experimental ORD values of (+)-limonene (chloroform, $22.4 \mathrm{mg} / \mathrm{ml}, 20.4{ }^{\circ} \mathrm{C}$ ) at four wavelengths (corrected by using the chloroform $[\alpha]_{\mathrm{D}}$ value $(113.8)$ of Rule and Chambers [40] which was itself corrected by a factor using the value of a homogenous sample of Wilson et al. [38]) and calculated ORD values (mpw1pw91/cc-pvdz, IEFPCM (methanol); In red: experimental; In black: mix 39:31:30 based on chemical shift prediction; In orange: mix 28:38:34 based on calculated free energies; In blue: mix 34:23:43 based on calculated enthalpies. a line broadening of $0.4 \mathrm{eV}$, the spectra in Fig. S6 A-I for conformer 1, Fig. S7 A-I for conformer 2, and Fig. S8 A-I for conformer 3 were obtained (supporting information). In addition, the calculated UV spectra for the conformers at different levels of theory are shown in the supporting information (Figs. S10-S12). It is important to note, that one level of theory was not able to deliver the longest wavelength absorption around $210 \mathrm{~nm}$ which was clearly observable in the experimental UV-Vis spectra. No wavelength shift was applied to allow an unbiased comparison. To obtain a robust computational result for comparison with experiment, we calculated the population average (39:31:30 for conformer 1, 2 and 3) of all levels of theory (Fig. 4A-I).

It is very important to measure samples with different concentrations since only then weak ECD signals can be observed (high concentration), and at the same time appropriately low noise levels are obtained (low concentration) for strong ECD signals. Two concentrations were measured in methanol and are depicted in Fig. 5A and B (UV: Fig. S13 A and B) to illustrate the concentration effects. We want to emphasize that this is not related to concentration effects due to aggregation. It is an important technical requirement. An ECD spectrum measured in ethanol is also presented (Fig. 5C; UV spectrum: Fig. S13C). Inspection of the spectra obtained at different concentrations indicates no aggregation effects that would shift the position of the cotton effect. In addition, the same results were observed for methanol and ethanol as solvent. From these results, we conclude that the solvent modelling presents no problem for our structural models when calculating UV-Vis and ECD spectra. A negative band can be seen around $195 \mathrm{~nm}$ and a 


\section{A: mpw1pw91/cc-pvdz, cost ${ }^{a}: 1$}
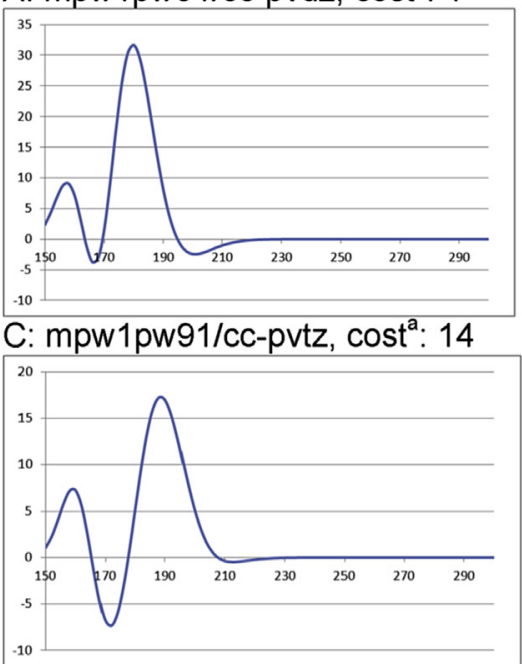

E: B3LYP/cc-pvdz, cost ${ }^{\mathrm{a}}: 1$

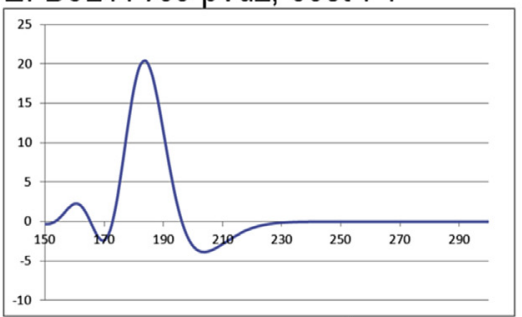

G: B3LYP/cc-pvtz, cost ${ }^{\mathrm{a}}: 14$
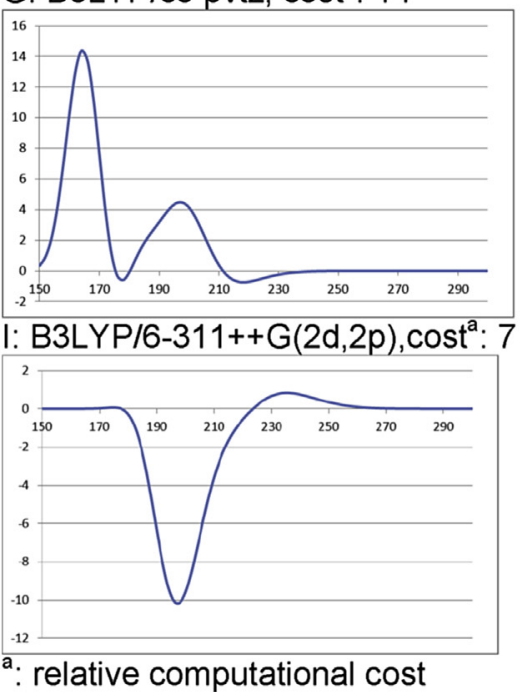

B: mpw1pw91/aug-cc-pvdz, cost ${ }^{\mathrm{a}}: 7$
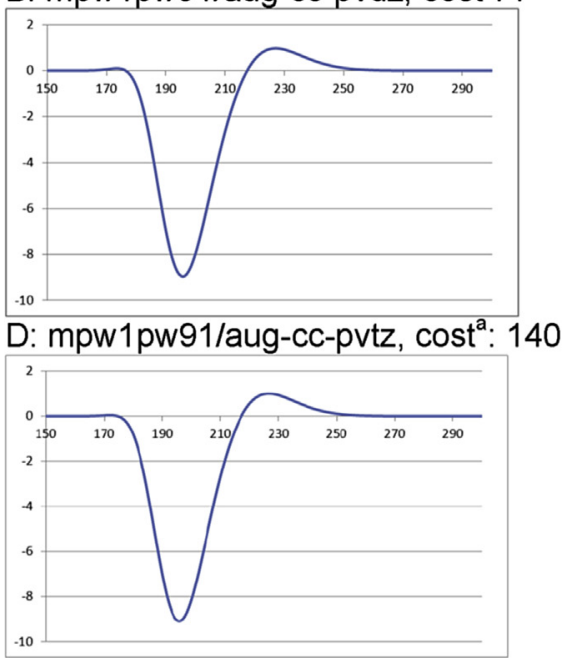

\section{F: B3LYP/aug-cc-pvdz, cost ${ }^{\mathrm{a}}: 7$}
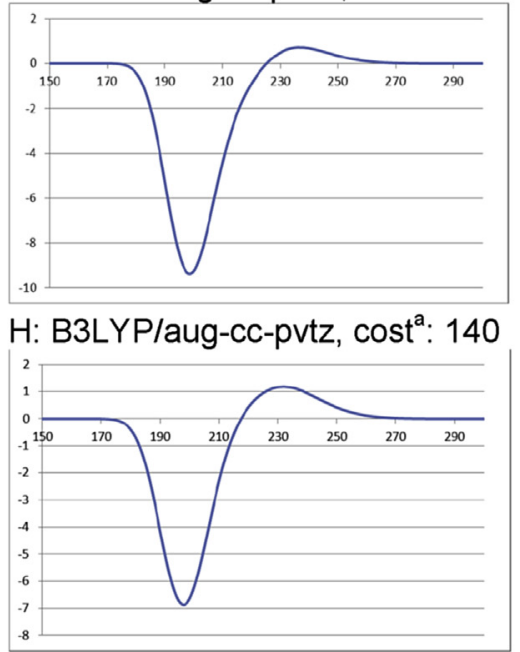

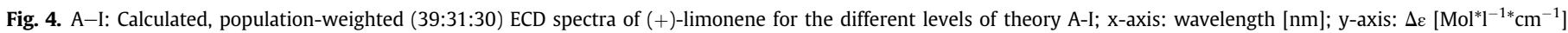
(IEFPCM using ethanol as solvent).

positive band around $210 \mathrm{~nm}$. These two decisive bands can be found in the calculated spectra (negative band at $196 \mathrm{~nm}$, positive band at $228 \mathrm{~nm}$ ) if Dunning's augmented basis sets or a Pople basis set with diffuse and polarization functions are applied (Fig. 4: levels of theory: B, D, F, H, and I). Importantly, spectra using the cc-pvdz and cc-pvtz basis sets would lead to a wrong absolute configuration assignment if a red shift would be applied to the calculated data of the theory levels A, C and E. Without a red-shift, level G would lead to a wrong AC assignment. These conclusions also hold for the different population mixes based on calculated energies (Fig. S9, supporting information). Inspection of the rotational strength (and also dipolar strength) does not help for the decision to apply a red-shift or not since similar ratios of the two bands were obtained for the different basis sets.

Applying basis sets with diffuse functions, we can safely assign the absolute configuration of (+)-limonene based on the comparison of the ECD bands.

\section{6. $V C D$}

A number of reports presented VCD spectra of limonene in the past $[27,41,42]$ also encompassing rarely measured areas of 
A

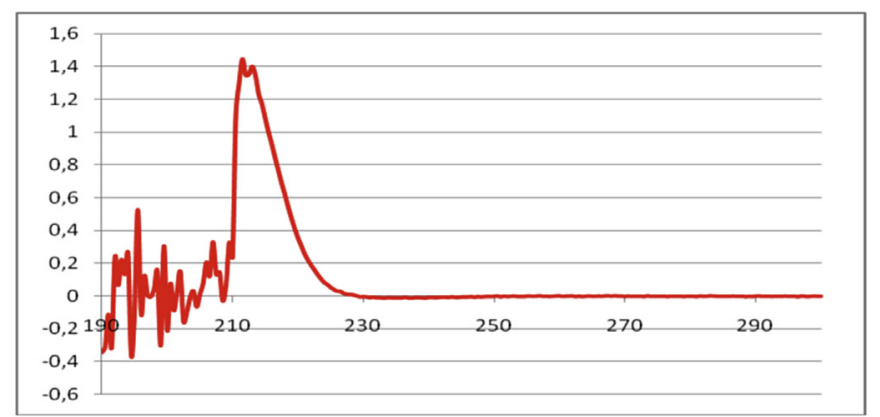

B

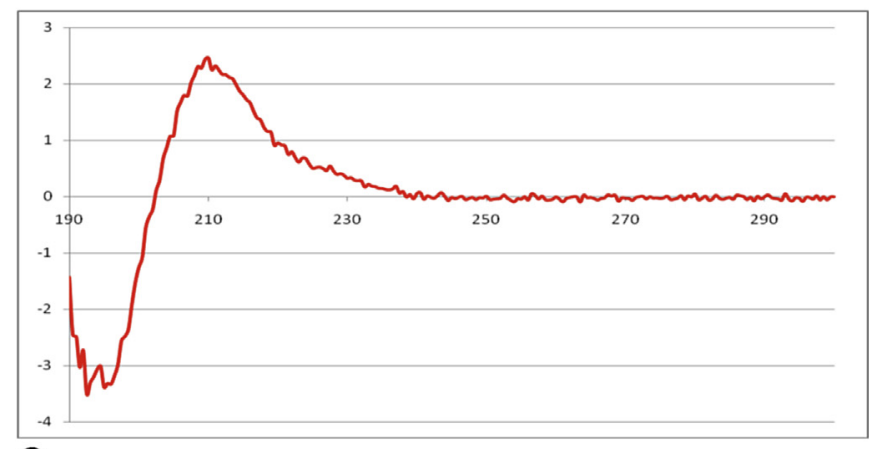

C

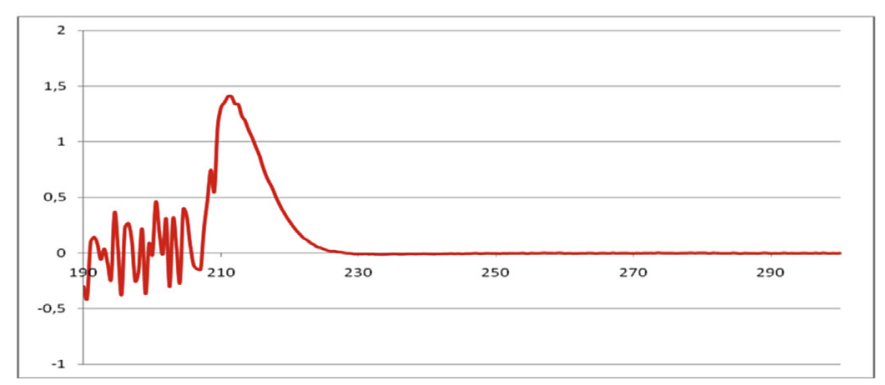

Fig. 5. A-C: Experimental ECD spectra of (+)-limonene in methanol or ethanol at different concentrations (on the x-axis: wavelengths in $\mathrm{nm}$, on the $\mathrm{y}$-axis $\Delta \varepsilon$ [ $\mathrm{Mol}^{*} \mathrm{I}^{-1 *} \mathrm{~cm}^{-1}$ ]; A: $2.7 \mathrm{mg} / \mathrm{ml}$ in methanol; B: $0.1 \mathrm{mg} / \mathrm{ml}$ in methanol; C: $2.4 \mathrm{mg} / \mathrm{ml}$ in ethanol.

wavenumbers (near infrared, $\mathrm{C}-\mathrm{H}$ stretching region). We measured the most often used range of wavenumbers between $1800 \mathrm{~cm}^{-1}$ and $1000 \mathrm{~cm}^{-1}$. Since pure limonene was used, the calculations used different solvents (chloroform and ethanol) to find a better mimic for limonene as solvent. In addition, different levels of theory were applied (Table 10) and the average of all calculated data was used to construct a calculated VCD spectrum using lorentzian line shapes with a half width at half height of $4 \mathrm{~cm}^{-1}$. The calculated and the experimental VCD spectra are shown in Figs. 6 and 7. Calculated spectra for each conformer at different levels of theory

\section{Table 10}

Calculated VCD spectra of (+)-limonene of the three conformers at different levels of theory.

\begin{tabular}{lll}
\hline Short notation & Theory level & Solvent (IEF-PCM) \\
\hline a & mpw1pw91/aug-cc-pvtz & Chloroform \\
b & mpw1pw91/cc-pvdz & Ethanol \\
c & mpw1pw91/aug-cc-pvtz & Ethanol \\
d & mpw1pw91/cc-pvdz & Chloroform \\
e & mpw1pw91/aug-cc-pvdz & Chloroform \\
\hline
\end{tabular}

can be found in the supporting information (Figs. S14-S17). It is important to note, that for these figures no shift in wavenumbers has been applied. It can be seen that there is no 1:1 match between the spectra concerning the band position, shape and intensity. Therefore, we propose the following qualitative analysis: we concentrate on the most significant stretch of bands in the experimental spectrum as being decisive for the conclusion if the calculated and experimental spectra match or if the inverted, calculated and the experimental spectrum matches, i.e. we focus only on the question of absolute configuration. This heavily depends on the correctness of a number of assumptions: the structural model represents one of the two enantiomers and no other molecule; the decisive bands are not inverted by matrix effects that have not been modelled; the position and sign of the decisive stretch of bands is correctly assigned.

In the case of limonene it is straightforward to select the decisive stretch of bands (i.e. large signal to noise ratio, at least 10; each band with a width of $10 \mathrm{~cm}^{-1}$ at minimum; peak to peak difference of $10 \mathrm{~cm}^{-1}$ at minimum; continues stretch of bands with isolated position): the bands around $1325 \mathrm{~cm}^{-1}, 1300 \mathrm{~cm}^{-1}, 1240 \mathrm{~cm}^{-1}$, and $1200 \mathrm{~cm}^{-1}$ can be classified as decisive, giving the pattern $+1-1+1-$ with intensities as medium/medium/strong/medium (Fig. 7B). Although the last two are quite near, the shape renders them well separated: a width and separation of at least $10 \mathrm{~cm}^{-1}$. However, since the accuracy of the calculated band positions is unknown and even sophisticated methods are not able to give precise answers [27], it is important to compare the whole stretch of possible decisive bands.

In the case of limonene we are therefore left with a stretch of four bands that we then call enantiodiscriminative. They are robustly calculated (different levels of theory, different solvents), and can be found at 1360,1320, 1280 and $1230 \mathrm{~cm}^{-1}$, Fig. 7A. From the match with experiment we safely conclude that $(+)$ limonene has $R$-configuration. All other interpretations of these bands do not fit with experiment (compare Figs. $7 \mathrm{~B}$ and 8, representing the calculated spectrum of $(S)$-limonene).

\section{Discussion}

\section{1. $O R D$}

Early attempts to correlate solvent effects on the optical rotation values with the refractive index [43] could already be rejected by Rule and Chambers [40]. Our results indicate that small solvent induced optical rotation changes could be explained by small population differences. However, an even likely explanation can be given based on the different calculated optical rotation values depending on the solvent used for modelling.

Lauricella et al. [44] tried to analyse the conformers of limonene by studying the temperature dependence of the optical rotation at $589 \mathrm{~nm}$. Interestingly, the authors explained the variation of rotivity in vacuo $\left(\Omega_{0}\right.$; the rotivity is defined as $\Omega=[\alpha] /\left(n^{2}+2\right)$, with $[\alpha]$ as measured optical rotation, and $n$ as refractive index of the sample) at different temperatures with a shift in the population mix: increasing the temperature resulted in decreased rotivities in vacuo. Using our population mix based on experimental ${ }^{13} \mathrm{C}$ resonances, we can support this view: increasing the temperature increases the populations of conformer 2 and 3 which have calculated negative optical rotation values so that the decrease of the experimental values with increased temperature is explained.

Pedersen et al. [45] investigated the temperature effects on ORD values of limonene using coupled cluster calculations. They compared the results for three conformers with experimental gas phase data at $355 \mathrm{~nm}$ and $633 \mathrm{~nm}$. The conformers are the three equatorial conformers that we have found. The calculated ORD 

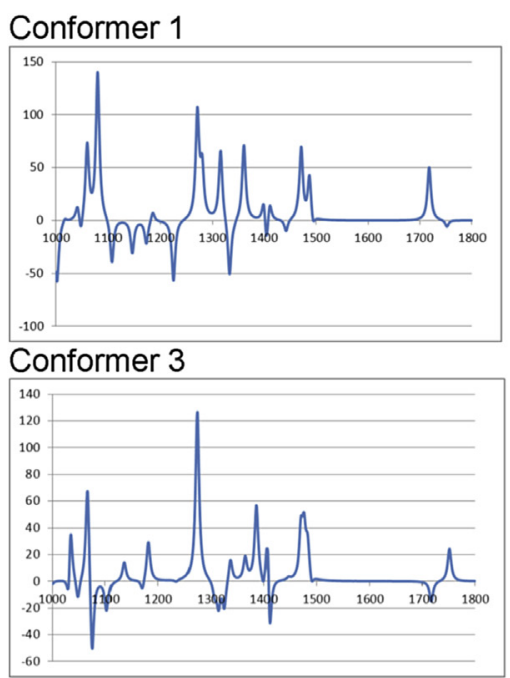

Conformer 2

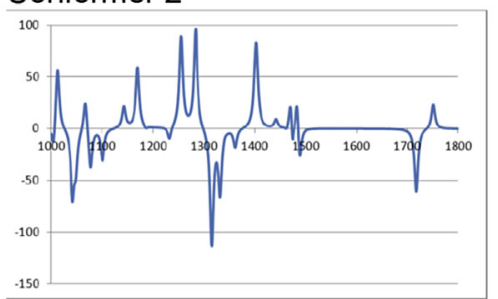

Population mix (39:31:30)

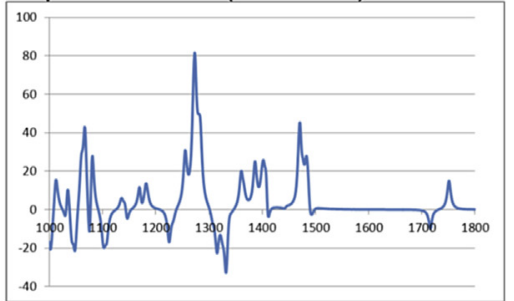

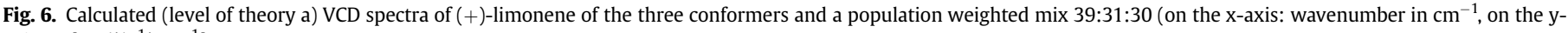
axis $\Delta \varepsilon\left[\mathrm{Mol}^{*} \mathrm{I}^{-1 *} \mathrm{~cm}^{-1}\right]$.

values (355 nm: 184.5; -1501.0; 543.2//633 nm: 11.6, -340.4, 112.0) differ from ours indicating that the level of theory and the incorporation of solvent affects the results. Interestingly, after assessing vibrational effects, the match with experiment was worse for CCSD compared to B3LYP: at $355 \mathrm{~nm}$ (-268.2 versus -311.1 ; exp: -315.5) [45]. Likewise, the incorporation of vibrational averaging for six conformational rigid molecules did not improve the prediction of the optical rotation, maybe since the calculations

\section{A}

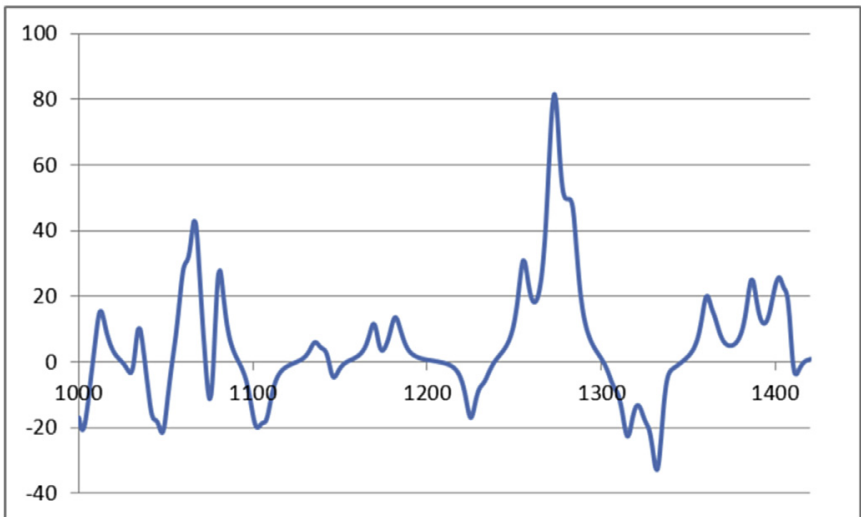

B

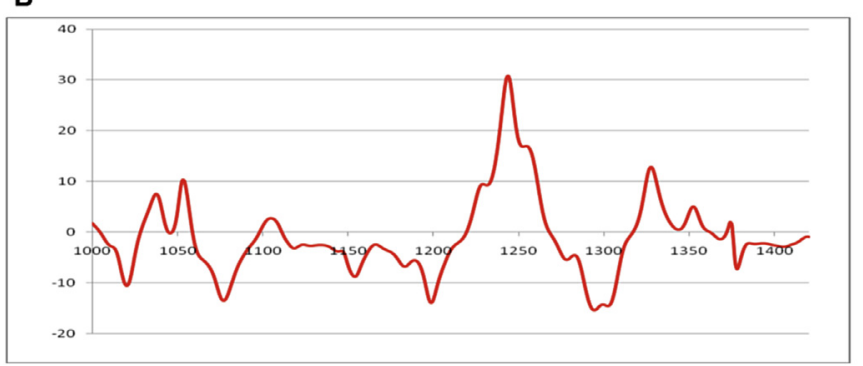

Fig. 7. A Calculated VCD spectrum of (+)-limonene (theory level a) using a populationweighted mix of 39:31:30 from $1420 \mathrm{~cm}^{-1}$ to $1000 \mathrm{~cm}^{-1}$ (on the x-axis: wavenumber in $\mathrm{cm}^{-1}$, on the $\mathrm{y}$-axis $\Delta \varepsilon$ [ $\left.\mathrm{Mol}^{*} \mathrm{I}^{-1 *} \mathrm{~cm}^{-1}\right]$. B: Experimental VCD spectrum of pure (+)-limonene: Region $1420 \mathrm{~cm}^{-1}$ to $1000 \mathrm{~cm}^{-1}$, on the $\mathrm{x}$-axis: wavenumber in $\mathrm{cm}^{-1}$, on the y-axis $\Delta \varepsilon\left[\mathrm{Mol}^{*} \mathrm{l}^{-1 *} \mathrm{~cm}^{-1}\right]$. were performed in vacuo and the experimental data were obtained in ethylcyclohexane [46]. Therefore, typical polarization effects of the solvent were neglected. In addition, Mort and Autschbach [46] concluded that the incorporation of vibrational effects on the ORD might not be sufficient to obtain accurate results as exemplified for methyloxirane and methylnorbornanone.

A publication on the gas phase optical rotation of limonene demonstrated that very small amounts of analyte can be measured: a detection limit of $20 \mathrm{ng}$ was determined [47]. The cavity-ringdown polarimetry was applied in a more recent study to measure the optical rotation of limonene in the gas phase at $355 \mathrm{~nm}$ and $633 \mathrm{~nm}$ [38]. The value of this technology is the absence of complicating solvent effects since their modelling is one of the critical points in the determination of absolute configuration using the comparison between calculated and measured chiroptical data. The archetypical problematic molecule, methyloxirane, could be measured, and gas-phase values compared with the condensed phase values. Since methyloxirane is regarded as rigid, complicating solvent effects on the population mix of conformers cannot be the reason for unsuccessful modelling of optical rotation values. One possible explanation is the inappropriate modelling of static versus dynamic distribution of electron density, which might explain the solvent dependence of the optical rotation of

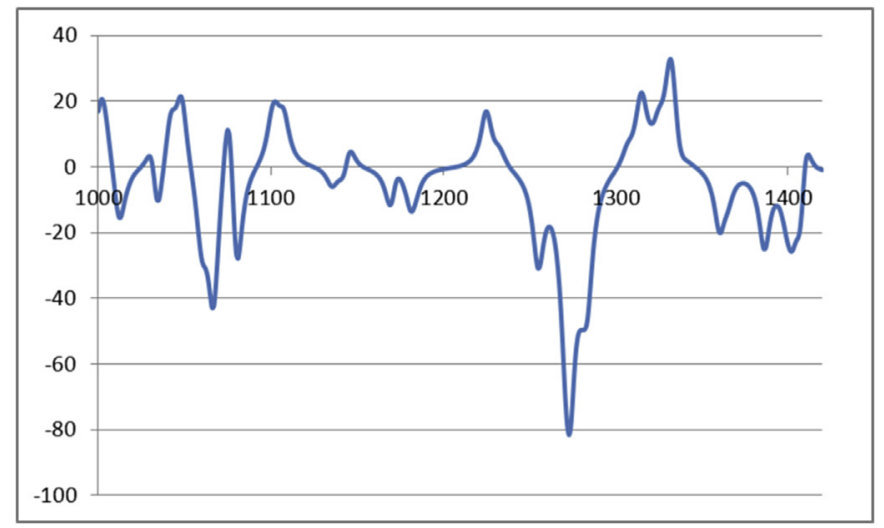

Fig. 8. Calculated VCD spectrum of (-)-(S)-limonene (theory level a; population mix of 31:39:30, on the x-axis: wavenumber in $\mathrm{cm}^{-1}$, on the y-axis $\Delta \varepsilon\left[\mathrm{Mol}^{*} \mathrm{l}^{-1 *} \mathrm{~cm}^{-1}\right]$. 
methyloxirane. To date, explanations are not conclusive.

Regarding the possible accuracy, two studies about the accuracy of ORD calculations are still valuable [48]. From comparisons between experiment and calculation, the authors concluded that on a $95 \%$ level of confidence, the correct absolute configuration could be predicted with confidence limits of $\pm 60 \mathrm{deg} /\left(\mathrm{dm}^{*}\left(\mathrm{~g} / \mathrm{cm}^{-3}\right)\right)$ [48]. In addition, Mennucci et al. [49] presented the successful application of the IEF-PCM approach to account for solvent effects based on electrostatics.

Experimental optical rotation values for limonene [38] gave values that are very similar to our calculated data assuming a 39:31:30 population mix. This parallels the data of Wilson et al. [38] since they obtained (B3LYP/cc-pvdz) at $355 \mathrm{~nm}$ a specific ORD of 270.3 , and at 633 a value of 76.3 , similar to our calculated data and the experimental values. However, the authors did not give detailed information how they obtained the three conformers, and which data they used for Boltzmann weighing of the conformers, nor they presented the individual ORD data for the conformers.

The importance of conformational effects on the ORD has been nicely described by Wiberg et al. [50] for a number of 3-substituted 1-butenes. Changing the temperature shifted the experimental ORD values which could be explained by a change in the conformer population, e.g. of 3-chloro-1-butene. However, the calculated ORD values of 3-chloro-1-butene were a factor of two larger compared to experiment, independent of the wavelength in the range from $589 \mathrm{~nm}$ to $250 \mathrm{~nm}$. The reason for this large discrepancy (e.g. $589 \mathrm{~nm}$ : -188.1 (calc), -73.5 (exp)) is not yet known, effects of vibrations have been investigated and were found to be present, but no conclusive explanation could be made. In a subsequent article, Wiberg et al. [51] analysed 1-butene, butane and related hydrocarbons. They realized that the choice of basis set is very important to accurately calculate ORD values. Diffuse functions should be used (augmented basis sets in the case of Dunning's correlation consistent basis sets such as cc-pvdz, aug-cc-pvdz). However, Rinderspacher and Schreiner [52] emphasized that the balanced nature of the basis set is the important aspect. Concerning the basis set, in the recent study of Hedegård et al. [53] it was shown that the aug-cc-pvdz basis set often led to significant deviations from the basis set limit. This is in contrast to the study of Grimme et al. [54]. Since the more recent study used a larger number of molecules, it is likely that the often recommended usage of basis sets with diffuse functions must still be handled with caution.

Concerning the level of theory, Crawford et al. [55] concluded that TD-DFT exhibits the cotton pole at higher wavelengths in the case of diffuse, Rydberg states when compared with CCSD calculation. Applying the mpw1pw91/cc-pvdz level of theory, the calculated ORD values of limonene at four wavelengths matched very well to the experimental values obtained in solution, with relative differences smaller than $5 \%$, and absolute deviations smaller than 5 . In addition, previous work on a minor conformer of strychnine indicated that the mpw1pw91/cc-pvdz level of theory is superior to the "working horse" of geometry optimization, i.e. B3LYP/6-31G(d) [17].

\section{2. $E C D$}

Very early, Scott and Wrixon [56] compiled ECD values of chiral olefins. However, inappropriate application of the octant rule led to a by chance correct assignment of limonene which was proven by Brint et al. [57]. The positive band of $(+)-R$-limonene (equals $D-$ limonene) around $210 \mathrm{~nm}$ measured in solution was assigned as $\pi$ to $\pi^{*}$ transition in agreement with the octant rule [56]. However, gas-phase measurements revealed the presence of two positive bands at $218 \mathrm{~nm}$ and $210 \mathrm{~nm}$ which are assumed to belong to the same transition [57]. These authors assigned this positive band to a $\pi$ to 3 s transition which is well known to show a blue-shift in solution. By chance, this positive band was taken erroneously by Scott and Wrixon [56] as $\pi$ to $\pi^{*}$ transition, which followed the octant rule. However, limonene follows an anti-octant rule, and Brint et al. [57] mention two other cases of this behaviour. Generally, the octant rule should not be applied any more for configurational purposes. However, it remains interesting to study this empirical rule, especially the reasons why it fails.

For calibration of the ECD bands, the accuracy with which the UV-Vis spectrum can be calculated is very important. As a good starting point, the study of Perpète et al. [58] can be used. They studied the $\lambda_{\max }$ values of dye molecules. Since focusing on a chemically related group of molecules will very often lead to a bias towards, in this case, higher accuracy of the calculated data, we have to be cautious and take the reported values as minimum error levels. A mean absolute error of $20.1 \mathrm{~nm}(0.113 \mathrm{eV})$ was found for a group of 86 naphtoquinone dyes in various solvents. Calculations slightly overestimated the $\lambda_{\max }$ values mean signed error of $-7.2 \mathrm{~nm}(0.039 \mathrm{eV})$. Linear regression could not improve the prediction significantly which can be interpreted that systematic error is not the main reason for the errors. The equation of the regression was: $\lambda_{\max }(\exp )=36.687+0.9053 \lambda_{\max }$ (calc). This led to a mean absolute error of $19.9 \mathrm{~nm}$, not significantly improved compared to the non-fitted value from above.

Autschbach et al. [59] proposed to use an overall damping of $0.2 \mathrm{eV}$ to simulate ECD spectra from calculated rotational strengths. In our work, we used several values, and decided to rely on a broadening value that allows the best match between the experimental and calculated UV-Vis bands. The latter point is also related to the accuracy with which the rotational strengths are calculated. Based on own results [17,60], we can assume differences of a factor of two between calculation and experiment concerning the rotational and dipolar strengths.

Conformational flexibility in solution often complicates the analysis since all populated conformers have to be taken into account. In this case solid state ECD could offer the possibility to calculate only the conformer which is found in the crystal. From the crystal structure obtained by x-ray, the ECD spectrum can be calculated and compared with experiment to assign the AC. However, as stated by Pescitelli [61], the influence of intermolecular interactions can hardly be predicted.

The main conclusion from our calculations is that non-diffuse basis sets do not show the important positive band around $220 \mathrm{~nm}$ so that a reliable AC determination is not possible. In contrast to the ORD results, for the ECD calculation it is imperative to use augmented Dunning's basis sets or Pople basis sets with diffuse functions for the correct prediction of the AC of limonene.

\section{3. $V C D$}

The importance of limonene as test molecule is demonstrated by a recent study of Rhee et al. [62]. They measured VCD spectra of 1.2-1.5 $\mathrm{M}$ solutions of limonene in $\mathrm{CCl}_{4}$ on a femtosecond time scale. The authors mention that this method could deliver molecular motion pictures of chiral processes. However, the methodology is far from being established and the necessarily high concentrations of analyte will be an obstacle in the future. Furthermore, based on calculated energies in vacuo (not specified which ones) Rhee et al. [62] calculated populations (29:17:54; MP2/6$\left.311++\mathrm{G}^{* *}\right)$ that differ largely from the ones of the present article (39:31:30). Since Rhee et al. [62] used these populations to simulate VCD spectra, the comparison with experimental data obtained in $\mathrm{CCl}_{4}$ as solvent is problematic. Surprisingly, QM/MM MD simulations with explicit solvent molecules gave similar VCD spectra with 
populations that resemble more the populations in the present article. Obviously, the good match to experiment was by chance with respect to the populations used.

A number of publications have used limonene as test molecule for VCD measurements (e.g. self-deconvolution of VCD spectra [42]; spectral standard) [63]. In addition, limonene has been used as test molecule for Raman optical activity spectroscopy [64]. The mostly applied mid-IR spectral range used for VCD ranges from $1800 \mathrm{~cm}^{-1}$ to $1000 \mathrm{~cm}^{-1}$ since skeleton vibrations near to the stereocenters are typically in this area. Polavarapu et al. [41] and Abbate et al. [65] studied the $\mathrm{CH}$ stretching region which is not often used in VCD analysis. Likewise, Singh and Keiderling [66] studied limonene using VCD in the $\mathrm{CH}$ stretching region and in the region of the important skeleton vibrations. They also started an interpretation of the spectra but this was based on the assumption of only two conformers which can be rejected by our data and other literature studies. The VCD bands that we have identified as decisive for the determination of the absolute configuration were also measured by Singh and Keiderling [66].

Although VCD spectra can be much more difficult to interpret for assigning the absolute configuration, a number of studies have been published in the area of natural product analysis (e.g. Gordillo-Román et al.) [67]. The very active research group of Avilés Moreno especially deals with the VCD analysis of natural products, such as limonene oxide [68] and carvone [69].

Partal Ureña et al. [70] studied limonene using the combination of IR, VCD and computations. Using experimental and calculated chemical shifts for the populations, we have determined different populations. Interestingly, with incorporation of a solvent model, our populations based on enthalpies are very similar to the populations calculated by Partal Ureña et al. [70] and Pedersen et al. [45], both calculated in vacuo.

A systematic approach for assigning the absolute configuration using VCD has been proposed by Sherer et al. [71]. However, even ibuprofen was a difficult case since additional information i.e. dimerization, had to be taken into account, not included by the systematic approach.

Vandenbussche et al. [72] presented a statistical validation of absolute configuration assignment using VCD for a series of quinolizine molecules. Polavarapu and Covington [73] presented dissymmetry factors as similarity measures for the analysis of VCD, ECD, and Raman optical activity spectra. The scientific community has not reached consensus in using a protocol to obtain a similarity factor when comparing experimental and calculated chiroptical spectra. Most often the harmonic approximation is used for the calculations so that anharmonic contributions are neglected which will be one of the reasons of mismatch between experiment and calculation. The importance of anharmonicity was shown by Cappelli et al. [74] for the test molecule formaldehyde. Current computer resources often limit a full anharmonic treatment of molecules larger than 30 atoms.

\subsection{Perspectives for chiroptical analysis}

A clear advantage of the ECD based configurational analysis is the low amount of compound that is needed for measurements. At the same time, aggregation effects will play the smallest role compared to the two other chiroptical methods, ORD and VCD. In this respect, ORD is intermediate but offers a remarkable number of advantages: it is easy to measure; the reliability can be increased by using short or long wavelengths; it is the only information regarding the absolute configuration of natural products isolated in the past, which are meanwhile decomposed and cannot be isolated again.

A further advantage for ECD is the possibility to use UV-Vis spectra as reference for the wavelength and intensity scale. Of course, VCD benefits in the same way from the comparison with the IR spectrum. However, the large concentrations needed due to the 10-100 fold lower intensity compared to ECD, renders the interpretation of the VCD spectrum very complicated due to low signal to noise ratios and possible aggregates. Especially the comparison with the calculated VCD spectrum for the assignment of the absolute configuration, will depend on the correct assumption of a single, solvated molecule since most often this represents the structural model for which the spectrum is calculated.

\section{Conclusions}

The present study about limonene gave important insights into the configurational analysis of small organic molecules using well known spectroscopic methods of NMR, VCD, UV-Vis, ECD, and ORD. To sum up, the main points are:

1. The current computational resources are able to identify the existing conformers in solution. However, in case of large and highly flexible compounds [75] experimental constraints are needed, mainly from NMR experiments, to narrow down the theoretical conformational space. In addition, the successful structural modelling of highly aggregating compounds depends on experimental information.

2. The populations should be determined with a combination of experimental and calculated data. Multi-standard methodologies for chemical shift calculations has been reviewed [76] and recently proposed [77]. Several systematic approaches in the field of structural analysis based on NMR data have been presented [78]. An early multiple regression analysis was presented by Sebag et al. [79] for tertiary amines. We term our approach "internal referencing" since resonances of the molecule itself are used for referencing. A similar approach has been presented by Andrews and Spivey [80]. They used reference molecules with known experimental chemical shifts which can be taken as fragments of the unknown molecule for which the shieldings are calculated. This approach differs from ours in that we directly take resonances of the unknown molecule for referencing. Obviously, our approach needs medium-sized molecules in order to have sufficient resonances for calibration/prediction. As an advantage, over-fitting can be easily discovered and corrected.

3. The chiroptical methods differ in a practical sense (amount needed, solubility etc.) but also in the response to the molecule under study leading to different signal to noise ratios.

4. Although all three chiroptical methods delivered the correct absolute configuration of limonene, it is a must to use at least two of them to assign the absolute configuration of an unknown compound.

The following procedure is proposed:

A. Achiral methods must agree on the same pair of enantiomers, i.e. all NMR and mass spectrometry data, as the two main methods, agree in the same structural model. In our experience, full assignment of resonances (including nitrogen), 2D NMR spectra fully interpreted (NOESY, COSY, HSQC, HMBC), proton and carbon 1D spectra with high resolution, homonuclear and heteronuclear scalar couplings (quantitative or qualitative), distances (qualitative from NOE intensities) are at present the complete requirements for a structural model of an unknown compound. In some cases, less information will also be sufficient. This is left to the NMR spectroscopist. Mass data must fit to the NMR derived structural model. 
B. All single steps of absolute configuration assignment must fulfil basic requirements.

1. The application of a number of reasonable levels of theory does not change the sign of the specific optical rotation, ORD, $\mathrm{ECD}$, and VCD data.

2. Absolute values of the experimental specific optical rotation at $589 \mathrm{~nm}$ should be larger than 60 . This range of only unreliably predictable optical rotations (AC assignments) will be narrowed down in a current research project about menthol isomers [81].

3. The experimental and calculated ORD curves at three or more wavelengths spanning at least $100 \mathrm{~nm}$ show no zero crossing.

4. The application of a reasonable wavelength shift (not more than $20 \mathrm{~nm}$ ) does not change the AC assignment based on ECD spectra.

5. There are no two or more enantiodiscriminative stretches of VCD bands with opposite AC assignment.

For limonene we could show that for the specific optical rotation at 589, the ORD values and VCD bands no sign change occurs if a number of reasonable levels of theory are applied. However, the ECD data cannot be used successfully since the AC assignment depends on the basis sets applied. In addition, the absolute value of the experimental specific optical rotation is larger than 60 , and the ORD curve with 4 wavelengths show no zero crossing so that all chiroptical methods except ECD (specific optical rotation > 60, ORD, and VCD) can be applied for the AC assignment.

\section{Experimental section}

$(R)-(+)$-limonene was purchased from Aldrich (>99.0\% purity).

\subsection{IR and VCD spectroscopy}

The VCD measurements were done with a VERTEX 80 FT-IR spectrometer equipped with a PMA 50 photoeleastic modulator and MCT-Detector (D313/B-A) (Bruker Optics). The VCD spectra were recorded with a 2-h data collection time at $4 \mathrm{~cm}^{-1}$ resolution using a cell with $\mathrm{BaF}_{2}$ windows. Spectra were measured at $22{ }^{\circ} \mathrm{C}$ as a neat solution with a path length of $0.1 \mathrm{~mm}$.

\subsection{ORD measurements}

Optical rotation measurements were performed using methanol and chloroform as solvents $(22.4 \mathrm{mg} / \mathrm{ml})$ at $20{ }^{\circ} \mathrm{C}$ (Polartronic-E polarimeter, Schmidt + Haensch GmbH\&Co).

\section{3. $E C D / U V-V i s$ spectroscopy}

ECD spectra were obtained with a Jasco J-810 spectrometer at $25{ }^{\circ} \mathrm{C}$. A cell with a pathlength of $1 \mathrm{~mm}$ was used, five scans were accumulated with a scan speed of $50 \mathrm{~nm} / \mathrm{min}, 0.5 \mathrm{~nm}$ pitch between $190 \mathrm{~nm}$ and $300 \mathrm{~nm}$.

\subsection{Computational details}

A model of $R$-limonene was built in GaussView 5.0. A conformational search using the BEST algorithm with the universal forcefield was performed using DISCOVERY Studio (Accelrys, 2009) [25]. DFT calculations were performed with Gaussian09 RevA.02 [26] on various levels of theory for geometry optimization, energy calculations, and spectroscopic properties. The ten lowest in energy excitation wavelengths and corresponding dipolar and rotatory strengths were calculated. Energies were obtained at $298.15 \mathrm{~K}$ and $1 \mathrm{~atm}$ of pressure. The lowest energy conformer was taken as reference, set to $0 \mathrm{kcal} / \mathrm{mol}$ and the resulting energy differences were used to calculate populations according to the Boltzmann distribution. In all calculations the Polarizable Continuum Model (PCM) using the integral equation formalism variant (IEFPCM) was used as solvent model.

\section{Acknowledgements}

We would like to thank Dr. Hans Abromeit for technical assistance and Prof. Gerhardt K. E. Scriba for helpful discussions. U.M.R. and F.R. would like to thank Prof. Griesinger (MPIBPC, NMR II), the Max-Planck-Society, and the DFG for support (FOR 934).

\section{Appendix A. Supplementary data}

Supplementary data related to this article can be found at http:// dx.doi.org/10.1016/j.molstruc.2015.10.061.

\section{References}

[1] G. Bifulco, P. Dambruoso, L. Gomez-Paloma, R. Riccio, Chem. Rev. 107 (2007) 3744-3779.

[2] S. Qiu, E. De Gussem, K.A. Therani, S. Sergeyev, P. Bultinck, W. Herrebout, J. Med. Chem. 56 (2013) 8903-8914.

[3] M. Pitzer, M. Kunitski, A.S. Johnson, T. Jahnke, H. Sann, F. Sturm, L.P.H. Schmidt, H. Schmidt-Böcking, R. Dörner, J. Stohner, J. Kiedrowski, M. Reggelin, S. Marquardt, A. Schießer, R. Berger, M.S. Schöffler, Science 341 (2013) 1096-1100.

[4] K.C. Nicolaou, S.A. Snyder, Angew. Chem. Int. Ed. 44 (2005) 1012-1044.

[5] M.E. Maier, Nat. Prod. Rep. 26 (2009) 1105-1124.

[6] W. Offermann, A. Mannschreck, Org. Magn. Reson 22 (1984) 355-363.

[7] G. Metha, S.R. Karra, J. Chem. Soc. Commun. (1991) 1367-1368.

[8] T. Hansson, B. Wickberg, J. Org. Chem. 57 (1992) 5370-5376.

[9] E.N. Pitsinos, N. Athinaios, V.P. Vidali, Org. Lett. 14 (2012) 4666-4669.

[10] A.F. Thomas, Y. Bessière, Nat. Prod. Rep. (1989) 291-309.

[11] W.A. Duetz, H. Bouwmeester, J.B. van Beilen, B. Witholt, Appl. Microbiol. Biotechnol. 61 (2003) 269-277.

[12] M.E.S. Lind, F. Himo, Angew. Chem. Int. Ed. 52 (2013) 4563-4567.

[13] K. Noack, Helv. Chim. Acta 52 (1969) 2501-2507.

[14] K. Kobayashi, Y. Asakawa, Y. Kikuchi, H. Toi, Y. Aoyama, J. Am. Chem. Soc. 115 (1993) 2648-2654.

[15] a M. Fukuji, Y. Kawagoe, Y. Nakano, A. Nakao, Molecules 18 (2013) 7035-7057;

b J. Aimi, K. Oya, A. Tsuda, T. Aida, Angew. Chem. Int. Ed. 46 (2007) 2031-2035; c M. Fujiki, Symmetry 6 (2014) 677-703.

[16] a L. Fu, Y. Zhang, Z.-H. Wei, H.-F. Wang, Chirality 26 (2014) 509-520; b : F.Wei, Y.-Y.Xu, Y.Guo, S.-L.Liu, H.-F.Wang, Chin. J. Chem. Phys. 2009, 22 592-600 c L. Velarde, X.-Y. Zhang, Z. Lu, A.G. Joly, Z. Wang, H.-F. Wang, J. Chem. Phys. 135 (2011) 241102.

[17] M. Schmidt, F. Reinscheid, H. Sun, H. Abromeit, G.K.E. Scriba, F.D. Sönnichsen, M. John, U.M. Reinscheid, Eur. J. Org. Chem. (2014) 1147-1150.

[18] F. Reinscheid, M. Schmidt, H. Abromeit, S. Liening, G.K.E. Scriba U.M. Reinscheid, J. Mol. Struct. (2015) (accepted).

[19] a F. Egidi, R. Russo, I. Carnimeo, A. D'Urso, G. Mancini, C. Cappelli, J. Phys. Chem. A 119 (2015) 5396-5404. E. C. Sherer, J. R. Cheeseman, R. T. Williamson, Org. Biomol. Chem. 2015, 13, 4169-4173; b P.L. Polavarapu, Chirality 20 (2008) 664-672.

[20] U.M. Reinscheid, J. Mol. Struct. 918 (2009) 14-18.

[21] H. Zhang, I.M. Oppel, M. Spiteller, K. Günther, G. Böhmler, S. Zühlke, Chirality 21 (2009) 271-275.

[22] E.D. Skakovskii, W.P. Kiselev, L.Y. Tychinskaya, A.G. Schutova, L.W. Gonsharova, E.W. Spiridowish, N.A. Bovdey, P.A. Kiselev, O.A. Gaidukevich, J. Appl. Spectrosc. 77 (2010) 329-334.

[23] F. Bohlmann, R. Zeisberg, E. Klein, Org. Magn. Reson 7 (1975) 426-432.

[24] P. Lesot, M. Sarfati, J. Courtieu, Chem. Eur. J. 9 (2003) 1724-1745.

[25] Accelrys, Discovery Studio, 2009.

[26] M.J. Frisch, et al., Gaussian 09 RevA.02, Gaussian Inc., Pittsburgh, PA, 2009.

[27] E. Debie, PhD thesis, 2008, Ghent University.

[28] C.O. da Silva, B. Mennucci, T. Vreven, J. Org. Chem. 69 (2004) 8161-8164.

[29] F.J. Devlin, P.J. Stephens, P. Besse, J. Org. Chem. 70 (2005) 2980-2993.

[30] B. Mennucci, M. Claps, A. Evidente, C. Rosini, J. Org. Chem. 72 (2007) 6680-6691.

[31] A.P. Scott, L. Radom, J. Phys. Chem. 100 (1996) 16502-16513.

[32] J.P. Merrick, D. Moran, L. Radom, J. Phys. Chem. A 111 (2007) 11683-11700.

[33] M.L. Laury, S.E. Boesch, I. Haken, P. Sinha, R.A. Wheeler, A.K. Wilson, J. Comput Chem. 32 (2011) 2339-2347.

[34] K.K. Irikura, R.D. Johnson III, R.N. Kacker, J. Phys. Chem. A 109 (2005) 8430-8437. 
[35] N.J.R. van Eikema Hommes, T. Clark, J. Mol. Model. 11 (2005) 175-185.

[36] A.J. Weldon, T.L. Vickrey, G.S. Tschumper, J. Phys. Chem. A 109 (2005) 11073-11079.

[37] M.A. Śmiałek, M.-J. Hubin-Franskin, J. Delwiche, D. Duflot, N.J. Mason, S. Vronning-Hoffmann, G.G.B. de Souza, A.M. Ferreira Rodrigues, F.N. Rodrigues, P. Limão-Vieira, PhysChemChemPhys 14 (2012) 2056-2064.

[38] S.M. Wilson, K.B. Wiberg, J.R. Cheeseman, M.J. Frisch, P.H. Vaccaro, J. Phys. Chem. A 109 (2005) 11752-11764.

[39] M.F. Leopold, W.W. Epstein, D.M. Grant, J. Am. Chem. Soc. 110 (1988) 616-617.

[40] H.G. Rule, A.R. Chambers, J. Chem. Soc. (1937) 145-153.

[41] P.L. Polavarapu, M. Diem, L.A. Nafie, J. Am. Chem. Soc. 102 (1980) 5449-5453.

[42] E.D. Lipp, L.A. Nafie, Appl. Spectrosc. 38 (1984) 774-778.

[43] K.L. Wolf, H. Volkmann, Z. Phys. Chem. B 3 (1929) 139-148.

[44] R. Lauricella, J. Kechayan, H. Bodot, J. Phys. Chem. 81 (1977) 542-547.

[45] T.B. Pedersen, J. Kongsted, T.D. Crawford, Chirality 21 (2009) E68-E75.

[46] B.C. Mort, J. Autschbach, ChemPhysChem 8 (2007) 605-616.

[47] J. Poirson, M. Vallet, F. Bretenaker, A. Le Floch, J.-Y. Thépot, Anal. Chem. 70 (1998) 4636-4639.

[48] P.J. Stephens, D.M. McCann, E. Butcus, E. Stoncius, J.R. Cheeseman, M.J. Frisch, J. Org. Chem. 69 (2004) 1948-1958. P. J. Stephens, D. M. McCann, J. R. Cheeseman, M. J. Frisch, Chirality 2005, 17, S52-S64.

[49] B. Mennucci, J. Tomasi, R. Cammi, J.R. Cheeseman, M.J. Frisch, F.J. Devlin, S. Gabriel, P.J. Stephens, J. Phys. Chem. A 106 (2002) 6102-6113.

[50] K.B. Wiberg, P.H. Vaccaro, J.R. Cheeseman, J. Am. Chem. Soc. 125 (2003) 1888-1896.

[51] K.B. Wiberg, Y.-G. Wang, P.H. Vaccaro, J.R. Cheeseman, G. Trucks, M.J. Frisch, J. Phys. Chem. A 108 (2004) 32-38.

[52] B.C. Rinderspacher, P.R. Schreiner, J. Phys. Chem. A 108 (2004) 2867-2870.

[53] E.D. Hedegård, F. Jensen, J. Kongsted, J. Chem. Theory Comput. 8 (2012) 4425-4433.

[54] S. Grimme, F. Furche, R. Ahlrichs, Chem. Phys. Lett. 361 (2002) 321-328.

[55] T.D. Crawford, L.S. Owens, M.C. Tam, P.R. Schreiner, J. Koch, J. Am. Chem. Soc. 127 (2005) 1368-1369.

[56] A.I. Scott, A.D. Wrixon, Tetrahedron (1970) 3695-3715.

[57] P. Brint, E. Meshulam, A. Gedanken, Chem. Phys. Lett. 109 (1984) 383-387.

[58] E.A. Perpète, C. Lambert, V. Wathelet, J. Preat, D. Jacqemin, Spectrochim. Acta A 68 (2007) 1326-1333.
[59] J. Autschbach, L. Jensen, G.C. Schatz, Y.C.E. Tse, M. Krykunov, J. Phys. Chem. A 110 (2006) 2461-2473.

[60] M. Schmidt, H. Sun, P. Rogne, G.K.E. Scriba, C. Griesinger, L.T. Kuhn, U.M. Reinscheid, J. Am. Chem. Soc. 134 (2012) 3080-3083.

[61] G. Pescitelli, Chirality 24 (2012) 718-724.

[62] J. Rhee, Y.-G. June, J.-S. Lee, K.-K. Lee, J.-H. Ha, Z.H. Kim, S.-J. Jeon, M. Cho, Nature 458 (2009) 310-313.

[63] C. Guo, R.D. Shah, R.K. Dukor, T.B. Freedman, X. Cao, L.A. Nafie, Vibr. Spectrosc. 42 (2006) 254-272.

[64] G.-S. Yu, T.B. Freedman, L.A. Nafie, J. Raman Spectrosc. 26 (1995) 733-743.

[65] S. Abbate, G. Longhi, L. Ricard, C. Bertucci, C. Rosini, P. Salvadori, A. Moscowitz, J. Am. Chem. Soc. 111 (1989) 836-840.

[66] R.D. Singh, T.A. Keiderling, J. Am. Chem. Soc. 103 (1981) 2387-2394.

[67] B. Gordillo-Román, J. Camacho-Ruiz, M.A. Bucio, P. Joseph-Nathan, Chirality 24 (2012) 147-154.

[68] J.R. Avilés Moreno, F. Partal Ureña, J.J. López González, PhysChemChemPhys 11 (2009) 2459-2467.

[69] J.R. Avilés Moreno, F. Partal Ureña, J.J. López González, Vibr. Spectrosc. 51 (2009) 318-325.

[70] F. Partal Ureña, J.R. Avilés Moreno, J.J. López González, Tetrahedron Asymmetry 20 (2009) 89-97.

[71] E.C. Sherer, C.H. Lee, J. Shpungin, J.F. Cuff, C. Da, R. Ball, R. Bach, A. Crespo, X. Gong, C.J. Welch, J. Med. Chem. 57 (2014) 477-494.

[72] J. Vandenbussche, P. Bultinck, A.K. Przybyl, W.A. Herrebout, J. Chem. Theory Comput. 9 (2013) 5504-5512.

[73] P.L. Polavarapu, C.L. Covington, Chirality 26 (2014) 539-552.

[74] C. Cappelli, S. Monti, G. Scalmani, V. Barone, J. Chem. Theory Comput. 6 (2010) 1660-1669.

[75] a H. Sun, E.J. d'Auvergne, U.M. Reinscheid, C.K.Z. Andrade, L.C. Dias, R.O. Rocha, C. Griesinger, Chem. Eur. J. 17 (2011) 1811-1817;

b U.M. Reinscheid, J. Farjon, P. Haberz, M. Blackledge, C. Griesinger, ChemBioChem 7 (2006) 287-296.

[76] M.W. Lodewyk, M.R. Siebert, D.J. Tantillo, Chem. Rev. 112 (2012) 1839-1862.

[77] A.M. Sarotti, S.C. Pellegrinet, J. Org. Chem. 77 (2012) 6059-6065.

[78] M. Elyashberg, K. Blinov, A. Williams, Magn. Reson. Chem. 47 (2009) 371-389.

[79] A.B. Sebag, D.A. Forsyth, M.A. Plante, J. Org. Chem. 66 (2001) 7967-7973.

[80] K.G. Andrews, A.C. Spivey, J. Org. Chem. 78 (2013) 11302-11317.

[81] F. Reinscheid, U.M. Reinscheid, J. Mol. Struct. (2015) (accepted). 